\title{
Embedding a topological group into its WAP-compactification
}

\author{
by \\ Stefano Ferri (Bogotá) and Jorge Galindo (Castellón)
}

\begin{abstract}
We prove that the topology of the additive group of the Banach space $c_{0}$ is not induced by weakly almost periodic functions or, what is the same, that this group cannot be represented as a group of isometries of a reflexive Banach space. We show, in contrast, that additive groups of Schwartz locally convex spaces are always representable as groups of isometries on some reflexive Banach space.
\end{abstract}

1. Introduction. A bounded continuous function $f: G \rightarrow \mathbb{C}$ defined on a topological group $G$ is said to be weakly almost periodic if the set of left translates $\left\{f_{x}: x \in G\right\}$ (where $f_{x}(y)=f(x y)$ ) is a weakly relatively compact subset of the Banach space $C_{b}(G)$ of all continuous bounded functions on $G$. The set $\operatorname{WAP}(G)$ of all weakly almost periodic functions on $G$ is a uniformly closed and translation invariant subalgebra of $C_{b}(G)$ and thus has the structure of a commutative $C^{*}$-algebra. The maximal ideal space of $\operatorname{WAP}(G)$ is known as the weakly almost periodic compactification of $G$ and will be denoted by $G^{\mathrm{WAP}}$. Thus $\operatorname{WAP}(G)$ is isomorphic (as a $C^{*}$-algebra) to $C\left(G^{\mathrm{WAP}}, \mathbb{C}\right)$. The term compactification is justified by the existence of a naturally defined continuous map $j: G \rightarrow G^{\mathrm{WAP}}$ with dense range. This function is one-to-one exactly when $\operatorname{WAP}(G)$ is a point separating subset of $C_{b}(G)$.

One of the main features of $G^{\mathrm{WAP}}$ is the existence of a product extending that of $G$ (that is, with $j(x y)=j(x) j(y)$ ). This multiplication makes $G^{\mathrm{WAP}}$ a semitopological semigroup in which the functions $y \mapsto g y$ are continuous on $G^{\mathrm{WAP}}$ for every $g \in G$. Hence $G^{\mathrm{WAP}}$ is what is known as a semigroup compactification (see [3, Chapter 2] for more on this subject).

Every compact semitopological group can be represented as a group of linear isometries of a reflexive Banach space, the latter equipped with the weak operator topology [23]. Recall here that the weak operator topology

2000 Mathematics Subject Classification: 43A60, 22A10, 46B20.

Key words and phrases: weakly almost periodic function, weakly almost periodic compactification, reflexively representable group, stable Banach space. 
on the space $B(E)$ of all continuous linear endomorphisms of a Banach space $E$ is defined, by analogy with the Hilbert space case, as the weak topology induced by the maps $\psi_{v, f}(T)=\langle f, T v\rangle$, where $v \in E, f \in E^{*}$, and $\langle\cdot, \cdot\rangle$ denotes the dual pairing between $E$ and $E^{*}$. Following again Hilbert space theory, the strong operator topology on $B(E)$ is defined as the weak topology defined by the maps $\rho_{v}(T)=T(v), v \in E$. It is well known that both the weak and the strong operator topologies coincide on the unitary group of a Hilbert space. Megrelishvili [19] proved that the same is true for the isometry group of any reflexive Banach space.

Putting all this together, and taking into account that every continuous function on a compact semitopological semigroup is weakly almost periodic, we have the following result.

TheOREM 1.1 ([19]). Let $G$ be a topological group. The following are equivalent:

(1) There is a topological isomorphism of $G$ into the isometry group of a reflexive Banach space with the strong operator topology.

(2) There is a semitopological semigroup isomorphism of $G$ into a compact semitopological semigroup.

(3) The algebra $\operatorname{WAP}(G)$ separates the identity e of $G$ from every closed subset of $G$ not containing e.

(4) The canonical morphism $\epsilon_{\mathrm{WAP}(G)}: G \rightarrow G^{\mathrm{WAP}}$ is a semitopological semigroup isomorphism (i.e. a homeomorphism and a semigroup isomorphism).

Because of property (1), groups satisfying these conditions are called reflexively representable.

Reflexive representability of a topological group, besides being an interesting property in its own right, can be regarded as an indicator of how much information about the topology of $G$ is encoded in $G^{\mathrm{WAP}}$ and as a measure of the richness of the algebra $\operatorname{WAP}(G)$.

When $G$ is locally compact, the left regular representation $\lambda(g) f=f_{g^{-1}}$, $g \in G, f \in L_{p}(G), p \geq 2$, establishes a topological isomorphism of $G$ into the isometry group of the reflexive Banach space $L_{p}(G)$. Locally compact groups are therefore reflexively representable. At the other extreme we find Megrelishvili's example [18] of a topological group with no nonconstant weakly almost periodic function; this is the group $H_{+}[0,1]$ of all orientation preserving homeomorphisms of $[0,1]$ with the topology of uniform convergence on compact subsets.

Locally compact groups belonging to the class [IN] (i.e. admitting a compact neighbourhood of the identity which is invariant under conjugation) have WAP-compactifications of formidable cardinality (see [5] and [6]), an indication of their sheer richness in WAP-functions. This led to the conjec- 
ture that all Abelian topological groups might be reflexively representable. We find however (see Section 2) an Abelian group (namely, the additive group of the Banach space $c_{0}$ ) that is not reflexively representable and thus disprove that conjecture. This answers, in the negative, questions posed by Megrelishvili in [20], [17] and [18] and by Glasner and Megrelishvili in [9]. In the last section we deal with Schwartz locally convex spaces. In [8] it was shown that Schwartz spaces need not be representable as groups of isometries of any Hilbert space. We see here in contrast that Schwartz spaces are always representable as groups of isometries on some reflexive Banach space.

1.1. Same problem, other algebras. The problem of establishing whether a topological group belonging to a particular class embeds into the semigroup compactification induced by a given admissible algebra is interesting for many different spaces of functions. Here we briefly sketch what is known concerning the above question when $\operatorname{WAP}(G)$ is replaced by other classical admissible algebras. We shall refer to the algebra $\operatorname{AP}(G)$ of almost periodic functions on $G$ and to the uniform closure of the Fourier-Stieltjes algebra $B(G)$ of $G$. We recall that a function $f: G \rightarrow \mathbb{C}$ is almost periodic provided that the set $\left\{f_{x}: x \in G\right\}$ is norm relatively compact in $C_{b}(G)$. The Fourier-Stieltjes algebra of $G$ is defined as the linear span of the set of positive-definite functions.

The compactification $G^{A P}$ induced by $\operatorname{AP}(G)$ is often called the Bohr compactification of $G$ and it is a compact topological group. This makes impossible for the homeomorphism $\epsilon_{A P}: G \rightarrow G^{A P}$ to be an embedding unless $G$ is precompact. The compactification $G^{B(G)}$ is Mayer's [16] Eberlein compactification. The morphism $\epsilon_{B(G)}: G \rightarrow G^{B(G)}$ is a homeomorphism exactly when $G$ is unitarily representable, that is to say, when $G$ is topologically isomorphic to the unitary group (= isometry group) of some Hilbert space. As we stated above, the left regular representation establishes a topological isomorphism of $G$ into the isometry group of $L_{p}(G)$. Taking $p=2$ we see that all locally compact groups are unitarily representable. There are some other interesting classes of unitarily representable groups, such as the additive groups of some Banach spaces, like $L_{p}(\mu), 1 \leq p \leq 2$, or free Abelian topological groups [24]. The question of unitary representability is treated in more detail in [8].

2. An Abelian group $G$ need not embed into $G^{\mathrm{WAP}}$. We begin with a few technical facts concerning $p$-limits which will be needed to present our main result. We recall that, given a set $\left\langle x_{d}\right\rangle_{d \in D}$ indexed by $D$ in a topological space $X$ and an ultrafilter $p$ on $D$, we say that $L$ is the $p$-limit of $\left\langle x_{d}\right\rangle_{d \in D}$ and write $p$-lim $x_{d}=L$ if for every neighbourhood $U$ of $L$ we have $\left\{d \in D: x_{d} \in U\right\} \in p$. In any topological space, if $p$-limits exist they 
are unique (see [10, Theorem 3.48]). Moreover, $X$ is compact if and only if, for every $D$, every indexed set $\left\langle x_{d}\right\rangle_{d \in D}$ and every ultrafilter $p$ on $D, p$-lim $x_{d}$ exists (see [10, Theorem 3.52]).

An important tool in dealing with weakly almost periodic functions is Grothendieck's double limit criterion, which says that a bounded, complexvalued function $f$ defined on $G$ is in $\operatorname{WAP}(G)$ if and only if the equality

$$
\lim _{m} \lim _{n} f\left(x_{n}+x_{m}\right)=\lim _{n} \lim _{m} f\left(x_{n}+x_{m}\right)
$$

holds for all sequences $\left\langle x_{n}\right\rangle$ and $\left\langle x_{m}\right\rangle$ in $G$ for which the above two limits exist (see [3, Theorem 2.3]). We need an extension of Grothendieck's double limit criterion to certain iterated $p$-limits of weakly almost periodic functions. We start with a definition.

Definition 2.1. We say that a permutation $\pi$ of the set $\{1, \ldots, n\}$ is a shuffle with cut $k$ (where $k$ is an integer $1 \leq k \leq n$ ), if, whenever $i$ and $j$ both belong to $\{1, \ldots, k\}$ or both belong to $\{k+1, \ldots, n\}$, then $i<j$ implies that $\pi(i)<\pi(j)$.

In other words, a shuffle with cut $k$ preserves the order of both the set $\{1, \ldots, k\}$ and the set $\{k+1, \ldots, n\}$. Shuffles are so called because they are exactly the permutations we can achieve with a usual riffle shuffle of a deck of $n$ cards.

The next lemma is [2, Lemma 9.19]. There attention was restricted to a distance function but the proof is exactly the same.

Lemma 2.2. Let $G$ be a topological group, $\phi \in \operatorname{WAP}(G)$ and fix integers $n>k \geq 1$. Let $\left\langle x_{i_{1}, \ldots, i_{k}}\right\rangle$ and $\left\langle y_{i_{k+1}, \ldots, i_{n}}\right\rangle$ be two multi-indexed sequences in $G$. Fix $n$ free ultrafilters $p_{1}, \ldots, p_{n}$ on $\mathbb{N}$. If $\pi$ is a shuffle with cut $k$, then

$$
\begin{aligned}
p_{1}-\lim _{i_{1}} \ldots p_{n}-\lim _{i_{n}} \phi\left(x_{i_{1}, \ldots, i_{k}}+y_{i_{k+1}, \ldots, i_{n}}\right) \\
\quad=p_{\pi(1)}-\lim _{i_{\pi(1)}} \ldots p_{\pi(n)}-\lim _{i_{\pi(n)}} \phi\left(x_{i_{1}, \ldots, i_{k}}+y_{i_{k+1}, \ldots, i_{n}}\right) .
\end{aligned}
$$

Proof. If $n=2$ (and $k=1$ ) the equality

$$
p_{1}-\lim _{i_{1}} p_{2}-\lim _{i_{2}} \phi\left(x_{i_{1}}+y_{i_{2}}\right)=p_{2}-\lim _{i_{2}} p_{1}-\lim _{i_{1}} \phi\left(x_{i_{1}}+y_{i_{2}}\right)
$$

follows directly from the definition of WAP-function. Since the set $\left\{\phi_{x}\right.$ : $x \in G\}$ is relatively weakly compact, there is a continuous bounded function $f_{0}: G \rightarrow \mathbb{C}$ with $p_{1}-\lim _{i_{1}} \phi_{x_{i_{1}}}=f_{0}$ (the limit is taken in the weak topology of $C(G)$ ). Let on the other hand $p_{2}-\lim _{i_{2}} y_{i_{2}}=y_{0} \in G^{\mathrm{WAP}}$. Now, since WAP-functions are exactly those extending continuously to $G^{\mathrm{WAP}}$, we have

$$
\begin{aligned}
p_{1}-\lim _{i_{1}} p_{2}-\lim _{i_{2}} \phi\left(x_{i_{1}}+y_{i_{2}}\right) & =p_{1}-\lim _{i_{1}} p_{2}-\lim _{i_{2}} \phi_{x_{i_{1}}}\left(y_{i_{2}}\right)=p_{1}-\lim _{i_{1}} \phi_{x_{i_{1}}}\left(y_{0}\right) \\
& =f_{0}\left(y_{0}\right)=p_{2}-\lim _{i_{2}} f_{0}\left(y_{i_{2}}\right)
\end{aligned}
$$




$$
\begin{aligned}
& =p_{2}-\lim _{i_{2}} p_{1}-\lim _{i_{1}} \phi_{x_{i_{1}}}\left(y_{i_{2}}\right) \\
& =p_{2}-\lim _{i_{2}} p_{1}-\lim _{i_{1}} \phi\left(x_{i_{1}}+y_{i_{2}}\right) .
\end{aligned}
$$

Once the case $n=2$ is proven, the proof follows by induction just as in [2, Lemma 9.19].

As stated in Theorem 1.1 a topological group $G$ embeds into $G^{\text {WAP }}$ if and only if it admits enough weakly almost periodic functions to separate its identity $e$ from every closed subset $C$ which does not contain $e$. The following lemma shows that, if this happens, then every neighbourhood of the identity contains another one which is defined by a single continuous weakly almost periodic function.

LEMMA 2.3. Let $G$ be a metrizable group equipped with a translation invariant metric $d$ and let $e$ denote its identity. If the embedding $w: G \rightarrow$ $G^{\mathrm{WAP}}$ is a homeomorphism, then for every $\varepsilon>0$ there exists a continuous weakly almost periodic function $\phi_{\varepsilon}$ and some $\delta_{\varepsilon}>0$ such that $\phi_{\varepsilon}(e)=0$ and, for every $x \in G$,

$$
\left|\phi_{\varepsilon}(x)\right|<\delta_{\varepsilon} \quad \text { implies } \quad d(x, e)<\varepsilon .
$$

Proof. For each $m \in \mathbb{N}$ consider a weakly almost periodic function $\phi_{m}$ such that $0=\phi_{m}(e) \notin \overline{\phi_{m}\left(C_{m}\right)}$ where $C_{m}$ denotes the closed set $\{x \in G$ : $d(x, e) \geq 1 / m\}$ and the closure is taken in $G$. The existence of such a function is guaranteed by Theorem 1.1.

Take $n$ such that $1 / n \leq \varepsilon$. The lemma then follows with $\phi_{\varepsilon}=\phi_{n}$ and $\delta_{\varepsilon}=\inf \left\{\left|\phi_{n}(x)\right|: x \in C_{n}\right\}$.

The proof of the following theorem is modelled on Raynaud's proof [22] of nonuniform embeddability of $c_{0}$ into $\ell_{2}$, as it appears in [2, Theorem 9.20].

THEOREM 2.4. The additive group of the Banach space $c_{0}$ is not reflexively representable.

Proof. Suppose towards a contradiction that $c_{0}$ embeds into its WAPcompactification and consider the weakly almost periodic function $\phi$ and the number $\delta>0$ determined by $\varepsilon=1 / 2$ in Lemma 2.3. Since $\phi$ is continuous, there is some $\alpha>0$ such that $\|x-y\|_{\infty}<\alpha$ implies $|\phi(x-y)|<\delta / 2$.

Fix $k$ such that $1 / k<\alpha$ and consider the vectors $s_{n} \in c_{0}$ with their first $n$ coordinates $1 / k$ and the rest 0 . Then $\left\|\sum_{j=1}^{2 k}(-1)^{j} s_{j}\right\|_{\infty}<\alpha$.

Clearly, the same inequality will hold for every sequence of indices $n_{1}<$ $\cdots<n_{2 k}$ :

$$
\left\|\sum_{j=1}^{k} s_{n_{2 j}}-\sum_{j=1}^{k} s_{n_{2 j-1}}\right\|_{\infty}<\alpha .
$$


Thus, for any $n_{1}, \ldots, n_{2 k}$ (with $n_{1}<\cdots<n_{2 k}$ ) we have

$$
\left|\phi\left(\sum_{j=1}^{k} s_{n_{2 j}}-\sum_{j=1}^{k} s_{n_{2 j-1}}\right)\right|<\frac{\delta}{2} .
$$

Taking $p$-limits along free ultrafilters $p_{1}, \ldots, p_{2 k}$, we get

$$
\left|p_{1}-\lim _{n_{1}} p_{2}-\lim _{n_{2}} \ldots p_{2 k}-\lim _{n_{2 k}} \phi\left(\sum_{j=1}^{k} s_{n_{2 j}}-\sum_{j=1}^{k} s_{n_{2 j-1}}\right)\right| \leq \frac{\delta}{2} .
$$

The permutation $1 \rightarrow 1,2 \rightarrow 3,3 \rightarrow 5, \ldots, k \rightarrow 2 k-1, k+1 \rightarrow 2$, $k+2 \rightarrow 4, \ldots, 2 k \rightarrow 2 k$ is a shuffle. By Lemma 2.2 (recall that $\phi$ is weakly almost periodic) the above limit equals

$$
\begin{aligned}
& p_{1}-\lim _{n_{1}} p_{3}-\lim _{n_{3}} p_{5}-\lim _{n_{5}} \ldots p_{2 k-1}-\lim _{n_{2 k-1}} p_{2}-\lim _{n_{2}} \ldots \\
& \ldots p_{2 k}-\lim _{n_{2 k}}\left|\phi\left(\sum_{j=1}^{k} s_{n_{2 j}}-\sum_{j=1}^{k} s_{n_{2 j-1}}\right)\right|,
\end{aligned}
$$

hence for large enough $n_{1}<n_{3}<\cdots<n_{2 k-1}<n_{2}<n_{4}<\cdots<n_{2 k}$ we have

$$
\left|\phi\left(\sum_{j=1}^{k} s_{n_{2 j}}-\sum_{j=1}^{k} s_{n_{2 j-1}}\right)\right|<\delta .
$$

The choice of $\phi$ and $\delta$ implies that

$$
\left\|\sum_{j=1}^{k} s_{n_{2 j}}-\sum_{j=1}^{k} s_{n_{2 j-1}}\right\|_{\infty}<\frac{1}{2} .
$$

But a moment's reflection taking into account that $n_{1}<n_{3}<\ldots<n_{2 k-1}<$ $n_{2}<n_{4}<\cdots<n_{2 k}$ shows that

$$
\left\|\sum_{j=1}^{k} s_{n_{2 j}}-\sum_{j=1}^{k} s_{n_{2 j-1}}\right\|_{\infty}=\left\|\sum_{j=1}^{k} s_{j}-\sum_{j=k+1}^{2 k} s_{j}\right\|_{\infty}=1 .
$$

This is the desired contradiction.

REMARK 2.5. Megrelishvili [20, 19, 18] and Megrelishvili and Glasner [9, Problem 6.11] asked whether the map $\epsilon_{\mathrm{WAP}}$ is a homeomorphism for every Abelian group. Megrelishvili specifically asked the same question for Banach spaces and, in particular, for $c_{0}$. This is natural, as it is well known that $c_{0}$ cannot be uniformly embedded into $\ell_{2}$, and therefore does not embed into its Eberlein compactification $G^{B(G)}$. Theorem 2.4 answers Megrelishvili's questions. Theorem 2.4 also answers Question 6.12 of [20] by showing that quotients of reflexively representable groups may fail to be reflexively representable. It suffices to observe that $c_{0}$, just as every separable Banach 
space, is a quotient of $\ell_{1}$ and that $\ell_{1}$ is reflexively representable. In fact, this shows that even a quotient of a unitarily representable space need not be reflexively representable.

REMARK 2.6. The proof of Theorem 2.4 could have been trivially modified to show that every spreading basis in a reflexively representable Banach space must be unconditional. We have chosen this simple statement to make the paper more accessible to nonspecialists in Banach space theory. For the concept of spreading and conditional basis we refer the reader to [2]. It should be added however that the proof of Theorem 2.4 cannot be applied to weakly sequentially complete, let alone reflexive, spaces, for in such a space every spreading basis is unconditional [15, Théorème 5].

\section{More reflexively representable, not unitarily representable} groups. Most of the known classes of reflexively representable groups are also classes of unitarily representable groups: locally compact groups, Hilbert spaces, $L_{p}(\mu)$ spaces with $1 \leq p \leq 2$, free Abelian topological groups. The only well-known source of reflexively representable groups that contains groups that are not unitarily representable is the class of stable Banach spaces introduced by Krivine and Maurey [14]. A Banach space $E$ is stable if for all bounded sequences $\left\langle x_{n}\right\rangle_{n}$ and $\left\langle y_{n}\right\rangle_{n}$ in $E$ and for all nontrivial ultrafilters $p_{1}$ and $p_{2}$, the following equality on the $p$-limits holds:

$$
p_{1}-\lim _{n} p_{2}-\lim _{m}\left\|x_{n}+y_{m}\right\|=p_{2}-\lim _{m} p_{1}-\lim _{n}\left\|x_{n}+y_{m}\right\| .
$$

The function $\|\cdot\| /(1+\|\cdot\|)$ on a stable Banach space is weakly almost periodic by Grothendieck's double limit criterion and thus stable Banach spaces are reflexively representable. This was first proved by Chaatit [4] without referring explicitly to weakly almost periodic functions. Among stable spaces we find all $L_{p}(\mu)$-spaces for $1 \leq p<\infty$. It is known on the other hand that $L_{p}(\mu)$ is not unitarily representable for $p>2$ (this depends on results of Schoenberg and Megrelishvili, see the discussion in [8]). For a proof of the reflexive representability of some $L_{p}(\mu)$-spaces that does not refer to the stability of the norm, see [17]. Using stable Banach spaces as a basis we show here that the Schwartz locally convex spaces constitute another class of reflexively representable topological groups with nonunitarily representable members. Schwartz spaces can be characterized as those locally convex vector spaces that can be represented as a subspace of a projective limit of Banach spaces $\left\{E_{i}, T_{i j}\right\}$ with $T_{i j}: E_{j} \rightarrow E_{i}$ compact for every $j>i$ (recall that a linear operator $T: E \rightarrow F$ between Banach spaces $E$ and $F$ is compact provided the image $T\left(B_{E}\right)$ of the unit ball $B_{E}$ of $E$ has compact closure in $F$ ). Let now $E$ denote a Banach space and equip its conjugate space $E^{*}$ with the topology $\tau_{\mathrm{k}}(E)$ of uniform convergence on compact 
subsets of $E$, the space $\left(E^{*}, \tau_{\mathrm{k}}(E)\right)$ is then a Schwartz space. Jarchow [11] and Randtke [21] independently proved that a locally convex space $E$ is a Schwartz space if and only if it is linearly homeomorphic to a subspace of some power of $\left(c_{0}, \tau_{\mathrm{k}}\left(\ell_{1}\right)\right)$, i.e., that the space $\left(c_{0}, \tau_{\mathrm{k}}\left(\ell_{1}\right)\right)$ is universal in the class of Schwartz spaces. We shall denote $\left(c_{0}, \tau_{\mathrm{k}}\left(\ell_{1}\right)\right)$ as $\mathcal{S}\left(c_{0}\right)\left(\tau_{\mathrm{k}}\left(\ell_{1}\right)\right.$ is the strongest locally convex Schwartz topology that is weaker than the norm topology of $c_{0}$ ).

While it is proved in [8] that $\mathcal{S}\left(c_{0}\right)$ is not unitarily representable, we prove below that it is reflexively representable. We shall derive this fact from the following factorization theorem of Fonf, Johnson, Plichko, and Shevchyk that uses Johnson's space $R$ defined in [12]. The Banach space $R$ is defined as the $\ell_{2}$-sum $R=\left(\sum_{n=1}^{\infty} M_{n}\right)_{\ell_{2}}$ of a suitable family of finite-dimensional spaces $M_{n}$ (see [7] or [12] for more details).

Theorem 3.1 (Theorem 2.1 of [7]). For each Banach space $X$ with the approximation property and for each compact subset $K$ of $X$, there is a compact one-to-one operator $T_{K}: R \rightarrow X$ with $T\left(B_{R}\right) \supset K$, where $B_{R}$ denotes the unit ball of $B_{R}$.

REMARK 3.2. We refer the nonspecialist reader to the monograph [25] where the meaning and significance of the approximation property is explained and $\ell_{p}$-sums $\left(\sum E_{n}\right)_{\ell_{p}}$ (and their duality properties) are introduced. We will content ourselves here with remarking that $\ell_{1}$ does have the approximation property.

COROLlary 3.3. The space $\mathcal{S}\left(c_{0}\right)$ and, a fortiori, every Schwartz space is reflexively representable.

Proof. The proof is as that of [8, Theorem 3.3]. Let $\mathcal{K}\left(\ell_{1}\right)$ denote any set that is cofinal in the family of all compact subsets of $\ell_{1}$ (ordered by inclusion). For each $K \in \mathcal{K}\left(\ell_{1}\right)$ we consider the compact operator $T_{K}: R_{K} \rightarrow$ $\ell_{1}$ defined on a copy $R_{K}$ of $R$ that is provided by Theorem 3.1.

Define now

$$
\Psi: \ell_{\infty} \rightarrow \prod_{K \in \mathcal{K}(E)} R_{K}^{*}
$$

as the product $\Psi=\prod_{K \in \mathcal{K}(E)} T_{K}^{*}$, where again $R_{K}^{*}$ represents a copy of $R^{*}$ :

This map is easily seen to be one-to-one. We now regard $\Psi$ as an operator defined on $\left(\ell_{\infty}, \tau_{\mathrm{k}}\left(\ell_{1}\right)\right)$. Since all $T_{K}$ 's are compact operators, $\Psi$ is continuous, and the covering property of the $T_{K}$ 's makes $\Psi$ an open mapping onto its image. We thus conclude that $\Psi$ is a linear homeomorphism of $\left(\ell_{\infty}, \tau_{\mathrm{k}}\left(\ell_{1}\right)\right)$ onto a subspace of $\prod_{K \in \mathcal{K}(E)} R_{K}^{*}$.

We see next that $\prod_{K \in \mathcal{K}(E)} R_{K}^{*}$ is reflexively representable. Each $R_{K}^{*}$ is linearly isometric to $\left(\sum_{n} M_{n}^{*}\right)_{\ell_{2}}$. Since each $M_{n}^{*}$ is finite-dimensional, it is stable. As the $\ell_{2}$-sum of stable Banach spaces is known to be stable (see 
[14, Théorème II.1]), we find that $R_{K}^{*}$ is a stable Banach space and thus reflexively representable. We observe finally that reflexive representability is preserved by arbitrary products: this follows from Theorem 1.1 because the composition of a projection with a weakly almost periodic function is again weakly almost periodic.

The space $\left(\ell_{\infty}, \tau_{\mathrm{k}}\left(\ell_{1}\right)\right)$, being isomorphic to a subspace of the product $\prod_{K \in \mathcal{K}(E)} R_{K}^{*}$, is therefore reflexively representable, since $\mathcal{S}\left(c_{0}\right)$ is a subspace of $\left(\ell_{\infty}, \tau_{\mathrm{k}}\left(\ell_{1}\right)\right)$ and is universal in the class of Schwartz spaces, and every Schwartz space is reflexively representable.

REMARK 3.4. Let us mention two recent results improving or generalizing Theorem 2.4. First, in a paper published shortly after the submission of the present article, Kalton [13] answered a long-standing question in nonlinear functional analysis by proving that $c_{0}$ does not admit a uniform embedding in any reflexive Banach space. This is a far reaching generalization of [22, Théorème 5.1] from which Theorem 2.4 follows if one uses the fact ([19]) that every reflexively representable group can be uniformly embedded in a reflexive Banach space. Later on, A. Berenstein, I. Ben-Yaacov and the first named author [1] proved that every reflexively representable topological group whose topology is induced by an invariant metric admits an equivalent distance that is both invariant and stable. Using this fact, nonreflexive representability of $c_{0}$ could be derived directly from [22, Théorème 5.1].

Acknowledgements. The first author was supported by a research grant of the Faculty of Sciences of Universidad de los Andes, and the second author by the Spanish Ministry of Science, grant MTM2008-04599/MTM and by Fundació Caixa Castelló-Bancaixa, grant P1.1B2008-26. The support is gratefully acknowledged. The first named author also wishes to thank all people of the Department of Mathematics of the University Jaume I for their hospitality.

\section{References}

[1] I. Ben Yaacov, A. Berenstein, and S. Ferri, Reflexive representability and stable metrics, preprint, http://arxiv.org/abs/0901.1003, 2009.

[2] Y. Benyamini and J. Lindenstrauss, Geometric Nonlinear Functional Analysis. Vol. 1, Amer. Math. Soc., Providence, RI, 2000.

[3] J. F. Berglund, H. D. Junghenn, and P. Milnes, Analysis on Semigroups, Wiley, New York, 1989.

[4] F. Chaatit, A representation of stable Banach spaces, Arch. Math. (Basel) 67 (1996), 59-69.

[5] S. Ferri and D. Strauss, A note on the $\mathcal{W} \mathcal{A P}$-compactification and the LUE-compactification of a topological group, Semigroup Forum 69 (2004), 87-101.

[6] M. Filali, On the actions of a locally compact group on some of its semigroup compactifications, Math. Proc. Cambridge Philos. Soc. 143 (2007), 25-39. 
[7] V. P. Fonf, W. B. Johnson, A. M. Plichko, and V. V. Shevchyk, Covering a compact set in a Banach space by an operator range of a Banach space with basis, Trans. Amer. Math. Soc. 358 (2006), 1421-1434.

[8] J. Galindo, On unitary representability of topological groups, Math Z., to appear.

[9] E. Glasner and M. Megrelishvili, New algebras of functions on topological groups arising from G-spaces, Fund. Math. 201 (2008), 1-51.

[10] N. Hindman and D. Strauss, Algebra in the Stone-Čech Compactification, de Gruyter Exp. Math. 27, de Gruyter, Berlin, 1998.

[11] H. Jarchow, Die Universalität des Raumes co für die Klasse der Schwartz-Räume, Math. Ann. 203 (1973), 211-214.

[12] W. B. Johnson, Factoring compact operators, Israel J. Math. 9 (1971), 337-345.

[13] N. J. Kalton, Coarse and uniform embeddings into reflexive spaces, Quart. J. Math. 58 (2007), 393-414.

[14] J.-L. Krivine et B. Maurey, Espaces de Banach stables, Israel J. Math. 39 (1981), 273-295.

[15] J.-T. Lapresté, Sur l'inconditionnalité des suites basiques écartables dans un espace de Banach, C. R. Acad. Sci. Paris Sér. A-B 284 (1977), A803-A805.

[16] M. Mayer, Strongly mixing groups, Semigroup Forum 54 (1997), 303-316.

[17] M. G. Megrelishvili, Reflexively but not unitarily representable topological groups, Topology Proc. 25 (2000), 615-625.

[18] - Every semitopological semigroup compactification of the group $H_{+}[0,1]$ is trivial, Semigroup Forum 63 (2001), 357-370.

[19] - Operator topologies and reflexive representability, in: Nuclear Groups and Lie Groups (Madrid, 1999), Res. Exp. Math. 24, Heldermann, Lemgo, 2001, 197-208.

[20] —, Topological transformation groups: selected topics, in: E. Pearl (ed.), Open Problems in Topology, Elsevier, 2007, 423-439.

[21] D. J. Randtke, A simple example of a universal Schwartz space, Proc. Amer. Math. Soc. 37 (1973), 185-188.

[22] Y. Raynaud, Espaces de Banach superstables, distances stables et homéomorphismes uniformes, Israel J. Math. 44 (1983), 33-52.

[23] A. I. Shtern, Compact semitopological semigroups and reflexive representability of topological groups, Russian J. Math. Phys. 2 (1994), 131-132.

[24] V. Uspenskiı̌, Unitary representability of free abelian topological groups, Appl. Gen. Topology 9 (2008), 197-204.

[25] P. Wojtaszczyk, Banach Spaces for Analysts, Cambridge Stud. Adv. Math. 25, Cambridge Univ. Press, Cambridge, 1991.

Departamento de Matemáticas

Universidad de los Andes

Carrera 1.a 19 A 10

Apartado Aéreo 4976

Bogotá D.C., Colombia

E-mail: stferri@uniandes.edu.co
Instituto de Matemáticas y Aplicaciones de Castellón (IMAC) Departamento de Matemáticas Universidad Jaume I E-12071 Castellón, Spain E-mail: jgalindo@mat.uji.es

Received April 11, 2007 